\title{
EL DESARROLLO DE LAS VILLAS Y PEQUEÑAS CIUDADES EN LOS ESPACIOS PERIFERICOS AGRARIOS. EL CASO GALLEGO
}

\author{
Román RODRIGUEZ
}

Departamento de Xeografia

Universidad de Santiago de Compostela

\begin{abstract}
Resumen: La inclusión de Galicia en el contexto económico occidental ha desencadenado un proceso de urbanización basado en la concentración espacial de la población y sus actividades, así como la difusión del modo de vida urbano. Este hecho supone una fase de transición hacia modelos de organización territorial donde las pequeñas ciudades y villas van a intervenir en el auge económico de las zonas agrarias más atrasadas.
\end{abstract}

Palabras clave: Desarrollo local, sistema urbano, modelo de organización territorial, red de asentamientos, "villa".

Abstract: The inclusion of Galicia in the occidental economic context has developed a process of urbanization based on the population spacial concentration and their activities, as well as the diffusion of the urban way of life. This fact supposes a chance towards patterns of territorial organization, where little cities and villas are involved in the economic progress of the more undeveloped rural areas.

Key words: Local development, Urban system, territorial management pattern, Villa. 


\section{SISTEMAS URBANOS Y DESARROLLO ECONOMICO}

En los modelos de desarrollo económico de las sociedades capitalistas la ordenación y el planeamiento del sistema de ciudades es una de sus premisas básicas. El papel de la red de asentamientos urbanos de un espacio, como instrumento creador y difusor de innovaciones, es crucial para asegurar el mantenimiento del desarrollo económico y, sobre todo, para lograr su expansión por el territorio (RIBAS y PIERA, 1974).

En el actual marco europeo frente a las áreas o ejes centrales, nos encontramos con otras de carácter dependiente y secundario, lo que conocemos como regiones periféricas. Para comprender la configuración de este sistema de especialización territorial de nivel continental, es necesario remontarse el siglo XVIII, pues tras la basculación del centro económico de la Europa mediterránea a la Europa noratlántica se gesta la organización urbana de la Europa de hoy (de VRIES, 1987). A partir de este momento las improntas de la Revolución Industrial y la era de la mecanización se insertan en un sistema preexistente que, indudablemente, modifica pero no sustituye en un grado excesivo. De este modo el proceso de concentración urbana posterior a 1820-1850 restaura las características jerárquicas implantadas durante el proceso de creación del sistema urbano europeo moderno (JOHNSTON, 1980)

Galicia, compartiendo su situación con otros espacios que conforman la actual periferia europea, pese a contar con una importante y antigua presencia de núcleos urbanos, no fue capaz de integrarse en el proceso de urbanización generado tras la Revolución Industrial, ni de formar una malla de ciudades racional que articulase de modo armónico el espacio regional.

Centrándonos en este último aspecto debemos decir que nuestra comunidad quedó durante mucho tiempo apartada y desconectada de los ejes de desarrollo, ya no sólo internacionales, sino también nacionales (PEREZ VILARIÑO, 1990). Sin embargo en las últimas décadas se está produciendo un cambio importante en la situación preexistente, pues tras la inmersión de lleno de Galicia en el contexto económico occidental (que según algunos autores como Souto González, se hace de modo forzado y controlado por fuerzas exteriores), se genera un claro proceso de urbanización del espacio gallego. Caracterizado tanto por la concentración espacial de la población y de las actividades agrarias, como por la difusión espacial de los valores, comportamientos y organización propia del mundo ciudadano (VINUESA ANGULO y VIDAL DOMINGUEZ, 1991). Es este un proceso inacabado, ya que se está produciendo en la actualidad, y extraordinariamente complejo, pues la dinámica urbana en las periferias sufre la coincidencia espacio-Galicia, por una parte, se aprecian los desajustes producidos por una tardía integración en las estructuras de los países 
centrales europeos y, por otra, se da una implantación acelerada de características urbanas de las fases postindustriales, como la metropolitarización en las periferias de las ciudades.

Retomando la idea inicial del paralelismo observable entre el grado de desarrollo económico y la existencia y buen funcionamiento de la malla de ciudades, se hace necesario profundizar en las particularidades de la red urbana gallega. Entendida ésta como una herramienta de intervención político-urbanística, y como instrumento difusor del desarrollo económico y bienestar de vida en una comunidad que, por sus singularidades (fuerte emigración, declive demográfico, base productiva agraria, rentas bajas, etc.), lo necesita. Por esto nos interesa conocer cómo se ha creado y articula el presente sistema de asentamientos, cómo nos encontramos en una fase de transición hacia nuevos modelos de organización territorial $\mathrm{y}$, sobre todo, cómo las villas y pequeñas ciudades van a intervenir en el auge socioeconómico de las áreas agrarias periféricas más atrasadas.

\section{DISPARIDAD TERRITORIAL GALLEGA}

Galicia como región doblemente periférica se inserta en el modelo clásico centro-periferia de J. Friedman por el cual las innovaciones y cambios surgidos en los espacios centrales se concentran primeramente en determinados puntos de los periféricos. En éstos se produce un proceso acumulativo de crecimiento y transformación de sus estructuras productivas internas, económicas y sociales, agravándose las desigualdades interterritoriales con un predominio de los efectos concentradores frente a los difusores (PRECEDO LEDO y RODRIGUEZ MARTINEZCONDE, 1991).

Esta visión encuentra un buen reflejo en la realidad gallega con la confrontación entre un eje de fuerte desarrollo y concentración de la población y las actividades económicas (el corredor litoral a lo largo de la Depresión Meridiana, de Ferrol a Vigo), y el resto de la comunidad dominada en su mayor parte por estructuras productivas de base agraria.

Desde el ámbito geográfico se han realizado importantes contribuciones para la comprensión de la organización espacial gallega. Esta se sintetiza en la existencia de una red bipolar alrededor de dos centros, uno meridional, Vigo, y otro septentrional, A Coruña. En torno a estos núcleos se forman dos áreas metropolitanas caracterizadas por la existencia de una estructura de asentamientos funcionalmente especializada de ámbito supramunicipal (municipios turísticos, industriales, residenciales,...), y 
complementadas por la presencia de otras dos ciudades de rango inferior (Pontevedra y Ferrol).

Las controversias surgidas en la definición, delimitación y funcionamiento de este tipo de formas de organización espacial no deben de desviarnos del fuerte proceso de periurbanización que encierran. Concentran más de un $50 \%$ de la población gallega siendo, ya no los municipios centrales sino los limítrofes, los que presentan en la actualidad los ritmos de crecimiento demográfico más elevado de la región. A su vez los flujos laborales cotidianos entre los espacios de producción y los residenciales generan movimientos pendulares de trabajadores de la industria y de los servicios. Cuentan, por otra parte, con una gran presencia de A.T.P. y de explotaciones agrarias muy intensivas y perfectamente introducidas en los círculos de mercado. Son también los sectores de mayor incremento del parque de viviendas que se basa, bien en el crecimiento de las villas próximas (Betanzos, Redondela,...), bien en la construcción de urbanizaciones de chalets y viviendas unifamiliares para primera o segunda residencia, o en la aparición de bloques residenciales a escasa distancia de la ciudad mayor (LOIS GONZALEZ, 1993). Estas grandes regiones urbanas difunden su influencia más allá de los límites teóricos de las áreas metropolitanas que delimitan, abarcando la mayor parte de Galicia y conectándose entre sí a través de la Autopista del Atlántico. En él se insertan numerosos asentamientos urbanos de pequeño tamaño (Vilagarcía, Padrón, Ordes, etc.). Y donde Santiago (aparte de sus funciones específicas) funciona como punto de conexión entre las dos grandes ciudades de la región.

Desde el punto de vista de la difusión del desarrollo económico, con la creación en 1962 de los Polos de Desarrollo de A Coruña y Vigo se favoreció la implantación de grandes complejos industriales por parte del I.N.I. y otros organismos y entidades de capital exógeno. De éstos. se esperaba la generación de procesos territoriales de difusión vertical y horizontal en gran parte del espacio gallego. No obstante, pese a la indudable importancia que esta política tendrá en su consolidación urbana, los objetivos para los que fueron creados no se lograron totalmente y, quizás exceptuando el caso de Citroen en Vigo, no se generaron movimientos relevantes de integración económica, produciéndose un agravamiento de las disparidades de base. Confirmándose así las limitaciones que las políticas regionales de desarrollo polarizado presentan para áreas periféricas al favorecer un crecimiento jerarquizado y no ser capaces de conseguir una reestructuración espacial capaz de difundir los efectos multiplicadores de los polos de crecimiento (VAZQUEZ BARQUERO, 1988).

Por lo que respecta a las ciudades que no forman parte del arco litoral, Lugo y Ourense, tenemos que decir que durante la mayor parte de su historia funcionaron como centros administrativos y comerciales de sus provincias, no interviniendo de forma destacada en el desarrollo territorial de su entorno. Tampoco la creación en 1975 de la 
Gran Area de Expansión Industrial que potenciaba un eje de equilibrio regional LugoMonforte-Ourense, supuso el despegue de estos núcleos como centros de transferencia de innovaciones y crecimiento económico en las áreas interiores más desfavorecidas.

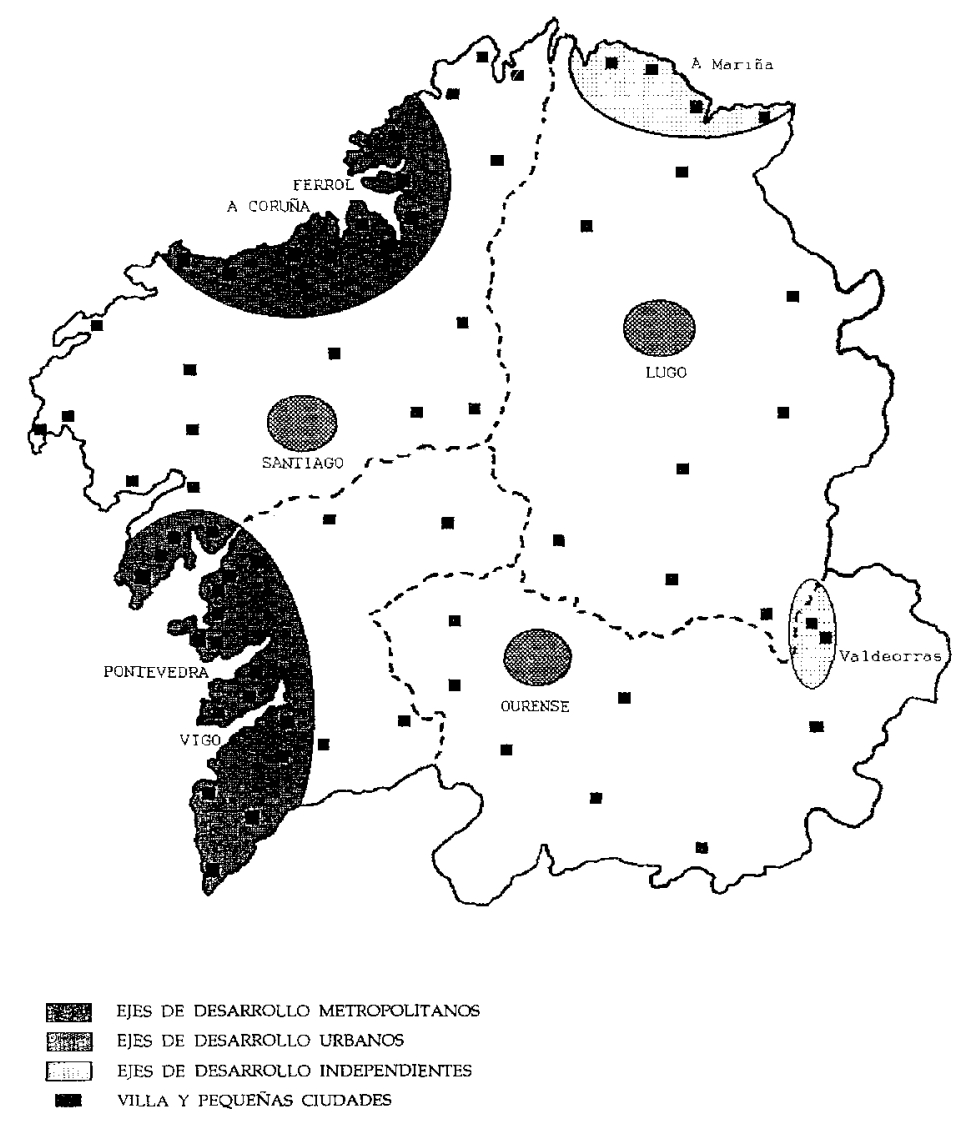

Figura 1.- Síntesis de los ejes de desarrollo de Galicia.

Si detallamos la escala de estudio nos encontramos con una amplia superficie agraria organizada en subsistemas comarcales definidos por la existencia de nodos urbanos de tamaño e historia muy diversa, y a los que denominamos villas, cabeceras comarcales o pequeñas ciudades. Estas centralizan gran parte de los flujos humanos y económicos de su espacio funcional, concentrando las actividades comerciales y de servicios públicos y privados que se destinan a satisfacer las necesidades inmediatas 
de la población del entorno. Los demás elementos de este tipo de subsistemas, núcleos complementarios más pequeños, asentamientos rurales y espacio agrario, configuran un claro modelo de organización del espacio de índole comarcal que podemos catalogar bien como comarcas agrarias, bien como comarcas funcionales presididas por una cabecera comarcal de marcada tendencia terciaria y en algunos casos, significativa presencia industrial.

El hecho de escoger la categoría de las pequeñas ciudades como referencia para nuestro estudio sobre la difusión del desarrollo económico en los espacios agrarios periféricos (ejemplificación en el caso galego) se sustenta tanto por la vocación de cubrir una laguna existente en los análisis que desde los diferentes ámbitos de las ciencias sociales se hacen sobre la organización territorial y económica y Galicia. Como por el interés de superar caducas visiones que, basándose en pautas macrodimensionales, consideran que en el campo galego no se han producido transformaciones ni ha tenido lugar un auge urbano tras la implantación de los modelos capitalistas e industriales en la región (SEQUEIROS TIZON, 1986). Estas, indudablemente, son menores de las deseadas, pero es innegable desde cualquier punto de vista, económico, cultural, de comportamientos,..., que en las últimas décadas el mundo rural gallego está sufriendo profundas mutaciones en sus estructuras internas como consecuencia del impacto que el proceso de urbanización, que afecta al conjunto del país, está jugando en él.

Por otra parte, nos interesa profundizar en el papel que las villas y pequeñas ciudades van a desempeñar como focos de aprovechamiento de los recursos propios de cada espacio comarcal, con vistas a desarrollo local conectado con los sistemas urbanos de mayor entidad.

\section{LAS PEQUEÑAS CIUDADES COMO AGENTES DEL DESARROLLO LOCAL}

Con unos volúmenes demográficos que varían entre los 2.000 y los $15.000 \mathrm{Hbt}$, y unas estructuras socio-profesionales de marcado carácter terciario (entre un $60-75 \%$ de la población activa), las villas en Galicia, al igual que sucede con las pequeñas ciudades en el resto de Europa, crecen desde los años sesenta en un proceso vinculado a los fuertes cambios económicos que se han registrado en el pais (LOIS GONZALEZ, 1993). Su consolidación dentro de la malla urbana tiene una gran importancia al servir de indiscutible enlace entre el mundo urbano y el rural en sentido estricto. Sirven de conexión y vehículo de contacto entre dos formas de organización que tienden a converger en la procura de una armonización territorial y social. 
Por ello, este tipo de núcleos cabeceras de comarca que gozan de hecho de una serie de ventajas competitivas (centralizan gran parte de las inversiones de su entorno), tienen que estar en el punto de mira de las políticas territoriales que desde la administración se encaminan al logro de un desarrollo regional, que como el caso de Galicia u otras autonomías, toman como base unidades comarcales.

Recogiendo la idea de la comarca como sistema y área de dependencia respecto a un núcleo central, cabe decir que existe una clara conexión de todos los elementos que la conforman. De modo que la modificación o los cambios que puede sufrir cada uno de ellos van a repercutir en la totalidad del conjunto. De este modo las comarcas y sus cabeceras configuran subsistemas dinámicos que evolucionan en función de los numerosos cambios que ha traido consigo el proceso de urbanización que se está viviendo. Así la villa se beneficia del desarrollo económico del espacio agrario dependiente (transformaciones ganaderas, especialización en producciones agrarias comercializables, aprovechamiento recursos forestales, etc.), pues los recursos monetarios que estas actividades generan repercuten de forma directa o indirecta en el crecimiento del núcleo central. A su vez, en algunas pequeñas ciudades se producen significativos procesos de industrialización generadores de empleo. De este modo se establece un modelo integrado de desarrollo económico de ámbito comarcal con una clara complementariedad de los elementos del sistema. En los espacios agrarios de Galicia se combina un doble modelo de crecimiento y consolidación de las villas y pequeñas ciudades basado en, por una parte, la modernización y capitalización de las actividades agrarias, lo que posibilita que una serie de asentamientos urbanos de reducido tamaño se especialicen en la oferta de bienes y servicios demandados por la agricultura en su proceso productivo. Por otra, el surgimiento y auge de procesos industriales en pequeños núcleos dispersos por el territorio gallego supone para la población de estas comarcas la posibilidad de acceder a nuevos mercados de trabajo sin necesidad de abandonar su lugar de residencia habitual.

En alguna comprobación empírica que se hizo sobre este fenómeno (RODRIGUEZ GONZALEZ, 1992), se aprecia la importancia que para un desarrollo económico integral del territorio tiene la confluencia entre los intereses de los agentes productivos rurales y urbanos. También se observó, y esto es extensible a la mayor parte del conjunto de villas, un cambio muy fuerte respecto a la funcionalidad que las actuales pequeñas ciudades tenían antes de la iniciación del proceso de urbanización, con la que hoy desempeñan. De este modo en las villas gallegas se detecta un doble ritmo de crecimiento poblacional y edificativo. Con anterioridad a 1960 el estancamiento y escaso vigor de estos índices contrasta con el de las tres últimas décadas de fuerte y rápido crecimiento. Obviamente esto implica que las funciones tradicionales que estos asentamientos cumplían durante la primera mitad de la centuria: mercados de intercambios agrarios periódicos, artesanado local, servicios de primerísima necesidad, etc., se transformen gracias al impacto que sobre las villas 
ejercen factores como la descentralización de toda una serie de servicios (sanitarios, educativos, financieros, etc.), el desarrollo y capitalización de las actividades agrícolas y el paso de antiguas actividades artesanales a actividades de corte imperial.

Estos cambios significan una adaptación a nuevas situaciones definidas por los diversos agentes humanos y económicos que se conjugaron en las últimas décadas en estos espacios y que contribuyen a fijar una nueva organización territorial en las áreas agrarias de Galicia: inversiones de capital procedentes de la emigración, cambio en las estructuras productivas agrarias, introducción de nuevas tecnologías o mejora de las infraestructuras.

\section{EL APROVECHAMIENTO DE LOS RECURSOS ENDOGENOS, CLAVE PARA EL DESARROLLO LOCAL}

Una de las características que definen a las regiones periféricas es el retraso con que son asumidas e interpretadas las políticas y las innovaciones encaminadas a mejorar el nivel económico y de bienestar de la sociedad. Así mientras en los países más industrializados durante los años 1950-70 se produce una desconcentración de la población y del empleo hacia los niveles más bajos de la jerarquía urbana, en el mismo período las regiones periféricas, como ya tuvimos ocasión de exponer, orientan sus políticas de desarrollo regional hacia una creciente concentración de las actividades productivas (Polos de Desarrollo).

Pero debido a la lentitud con la que incidió el proceso de industrialización en todo el territorio nacional se produce una superposición de estructuras productivas entre la tendencia concentradora de las actividades industriales en un número restringido de grandes ciudades, y el fortalecimiento de experiencias locales de industrialización vinculadas a los recursos endógenos (VAZQUEZ BARQUERO, 1999).

En Galicia se consolida, de este modo, un considerable sector productivo basado en empresas localizadas en tradicionales villas agrarias que no se debe interpretar como un proceso de difusión económica promovido desde las áreas centrales del sistema económico regional, a través de mecanismos de descentralización productiva y funcional, tras la crisis de los setenta. No coincidimos así con las lecturas que algunos investigadores como Rodríguez Galdo (1992) realizan sobre los procesos de industrialización endógenos en los espacios rurales de Galicia, al basarse en la extrapolarización de fenómenos registrados en las áreas centrales a las áreas periféricas. 
En el caso de Galicia la creciente importancia de la industria en las villas se vincula a iniciativas empresariales locales gestadas en la sustitución y evolución de tradicionales actividades artesanales que se adaptan con éxito a la nueva coyuntura basándose en los recursos humanos, físicos y financieros de sus espacios de referencia. $\mathrm{O}$ bien de pequeñas industrias que se desarrollan de modo complementario a las actividades agropecuarias ofreciéndoles "imputs" para su proceso productivo o transformando su producción final.

Tomando como referencia las pequeñas ciudades francesas para ejemplificar estas diferencias entre las regiones centrales y las periféricas, podemos decir que aquí la descentralización industrial tras "L'effect Buron" supuso la redistribución por el medio rural de más de 600.000 empleos industriales directos antes de la crisis del 74 (BEAUJEU-GARNIER, 1983). Por lo tanto y con posterioridad a la implantación de estas unidades industriales en asentamientos anteriores de base agraria, se configura una mullida red de pequeñas ciudades que fijan mano de obra salida del empleo rural en un modelo de reindustrialización difusa.

En Galicia como hemos subrayado, este proceso presenta unas características bien diferenciadas ya que la mayoría de las empresas industriales diseminadas por el medio rural se basan en el aprovechamiento, complementariedad y transformación de los recursos endógenos de cada territorio. Este hecho es uno de los que mejor expresa el papel de las pequeñas ciudades como partícipes del desarrollo local. Pues la explotación directa de las potencialidades de su entorno supone, por una parte, alejarse de una de las premisas de las regiones periféricas (fuente de mano de obra y de materias primas), y por otra, la generación de procesos de integración económica, verticales y horizontales, que sirven para crear empleo, frenar la emigración y el despoblamiento del rural y, en general, aumentar la calidad de vida de la población.

En la búsqueda de un correcto desarrollo endógeno es necesario tener en cuenta un factor que, en muchas ocasiones se escapa de la mayoría de los análisis que se hacen sobre el tema, como son los cambios culturales de valores y de comportamientos que introduce el proceso de urbanización y sin los que sería muy difícil que una comunidad se inserte y controle el nuevo proceso productivo. En primer lugar, un aprovechamiento endógeno implica una adaptación de las iniciativas empresariales al tejido social para evitar la generación de procesos traumáticos en el cambio de las estructuras productivas (que sí se puede producir en las industrias de enclave e implantación). En segundo lugar, es necesaria una mentalidad reinversora de los beneficios por parte del empresario local, posibilitando, en buenas coyunturas de crecimiento, que talleres puramente artesanales a principios de los sesenta se modernicen y tranformen, y hoy sean medianas empresas con niveles de facturación respetables (RODRIGUEZ GONZALEZ, 1992). En tercer lugar es lógico que las conexiones recursos-empresas se complementen (en los espacios de desarrollo ganadero se localizan fábricas de pienso, 
transformadoras de lácteos, talleres de maquinaria agrícola,...; en las áreas vitícolas, fábricas de envases de vidrio, embotelladoras,...; en las de riqueza mineral, empresas transformadoras del material extraído, distribuidoras,...). Aunque esto no es obstáculo para la consolidación de otro tipo de ramas fabriles que no emplean de modo directo las materias primas del territorio (textil, muebles, carpintería metálica,...), pero que siguen caracterizándose por un control de la iniciativa por parte de la comunidad local.

Otro factor que incide directamente en el esquema anterior es el papel de los emigrantes que retornan de las áreas centrales y se reintegran en la vida local eligiendo como nueva residencia ya no la parroquia de origen sino la cabecera comarcal más próxima. Aparte del incremento demográfico y la rehabilitación económica que supone este proceso (inversiones en pequeños negocios, nuevas empresas, construcción, etc.), el aporte tanto de capital como de capacidad innovadora y mentalidad productiva adquirida en los países de destino es un importante empujón al proceso de urbanización, contribuyendo a la configuración de las cabeceras como pequeñas ciudades impulsoras de desarrollo en su entorno.

\section{LOS MODELOS DE DESARROLLO LOCAL EN LAS PEQUEÑAS CIUDADES DE GALICIA}

La finalidad de este ejercicio es doble. En primer lugar nos permitirá observar de forma directa cómo el discurso teórico anteriormente expuesto se basa en una reflexión sobre los actuales procesos espaciales, sociales y económicos de la región, plasmándose aquí con cifras y datos concretos. En segundo término se analizará cómo las riquezas y potencialidades de cada territorio van a generar distintos mecanismos de respuesta en los individuos que se dedican a explotarlas, y cómo éstos van a repercutir de manera directa en la dinámica de las pequeñas ciudades dentro de un contexto de urbanización creciente de la sociedad gallega.

Por otra parte interesa centrarnos en dos modelos básicos de desarrollo local. En primer lugar, aquellas villas cabeceras de comarca de fuerte presencia agrícola/ganadera, en las que las mutaciones de las estructuras productivas de las diferentes actividades primarias supone el fortalecimiento de los núcleos rectores de cada espacio comarcal. En segundo, aquellas villas que, aún pudiendo ser partícipes del modelo anterior, deben su crecimiento a la consolidación de procesos industriales endógenos, bien en función de los recursos físicos locales, bien en la sustitución de estructuras artesanales por estructuras de corte empresarial. 
A tal efecto individualizaremos varios modelos de desarrollo para las villas. Por una parte, villas basadas en un modelo agropecuario, pudiendo tomar como ejemplo municipios como Negreira y Santa Comba para la actividad ganadera, y Ribadavia y A Rúa para el aprovechamiento de la vid. Por otra, villas basadas en modelo de desarrollo de base industrial, tomando O Barco y As Pontes como ejemplos de villas asociadas a la explotación minera y Lalín y Sarria como representantes de modelos industriales de base diversificada.

El encauzamiento y concentración en el aprovechamiento de los recursos endógenos por parte de estos asentamientos se traduce en una importante vitalidad demográfica. Tanto es así que las pequeñas ciudades se convierten en uno de los núcleos (no confundir con municipios) de mayor dinamismo demográfico de la comunidad gallega, igualando, o superando en tasas de crecimiento relativo, a las siete ciudades principales y a los núcleos periurbanos. Aunque su efectivo humano total puede ser modesto (nunca superan los $15.000 \mathrm{Hbt}$.), su nivel de incremento demográfico a lo largo del presente siglo supera, para los ejemplos seleccionados, un $430 \%$ sobre su población inicial, siendo, para los que cuentan con modelos de desarrollo endógeno más acertados o una mayor riqueza industrial (Lalín $695 \%$, As Pontes $642 \%$ ) superiores a los de las ciudades de Vigo (602\%) o A Coruña $(468 \%$ ). Y, por supuesto, muy superiores a la media gallega de un $136 \%$.

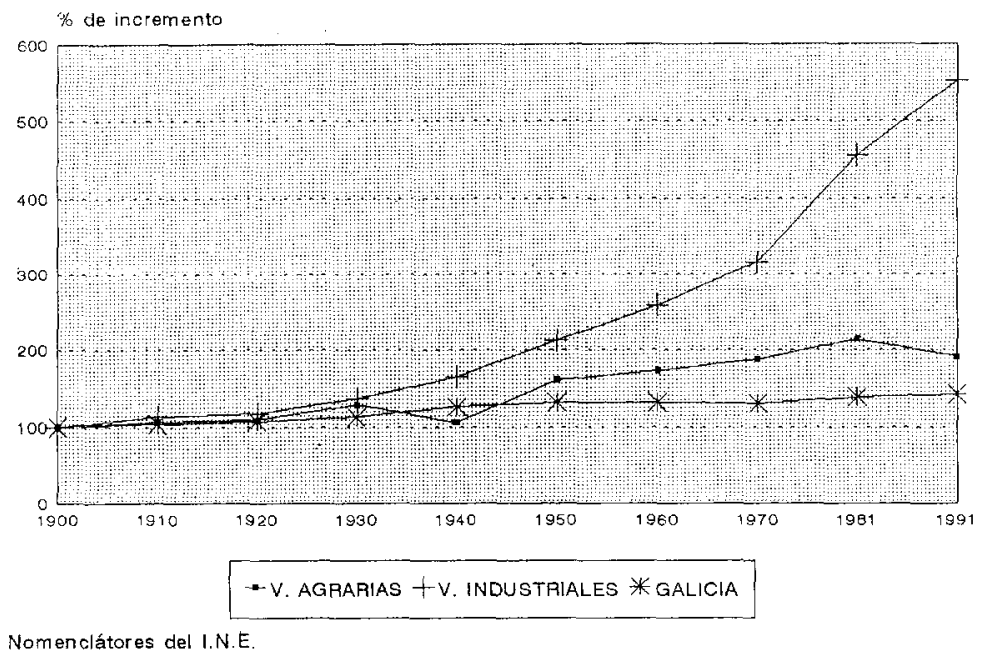

Figura 2.- Evolución demográfica comparada, villas agrarias-villas industriales de Galicia. 
Si individualizamos los ritmos de crecimiento demográfico de los ejemplos seleccionados y los comparamos con la secuencia total del territorio gallego (Fig. 2) podemos establecer de principio una compartimentación en tres fases bien diferenciadas. En un primer momento, hasta 1940, la evolución que presentan tanto las villas del modelo agrario como aquellas del modelo industrial es muy parejo entre sí y también con el conjunto de Galicia. Es esta una fase de anquilosamiento de los procesos de modernización de las estructuras productivas que impide la aparición de centros de desarroollo local vinculados a las potencialidades de cada entorno.

Durante los años cincuenta y sesenta se advierte una fase de transición en la cual los índices comienzan a diverger. El incremento porcentual de las villas comienza a superar en estas fechas al total de Galicia y, muy especialmente, en aquéllas vinculadas a diversos fenómenos de industrialización endógena, en función de una serie de cambios estructurales registrados en la economía española. Estos cambios se pueden concretar en las consecuencias que el aperturismo del estado va a acarrear en el conjunto de las estructuras productivas, sociales y territoriales. En este momento de adaptación se inicia un fenómeno de despoblamiento en las áreas rurales de los espacios periféricos del sistema, y es por ello que en Galicia se materializa en un despoblamiento masivo de los espacios agrarios que se acentuará en la fase posterior.

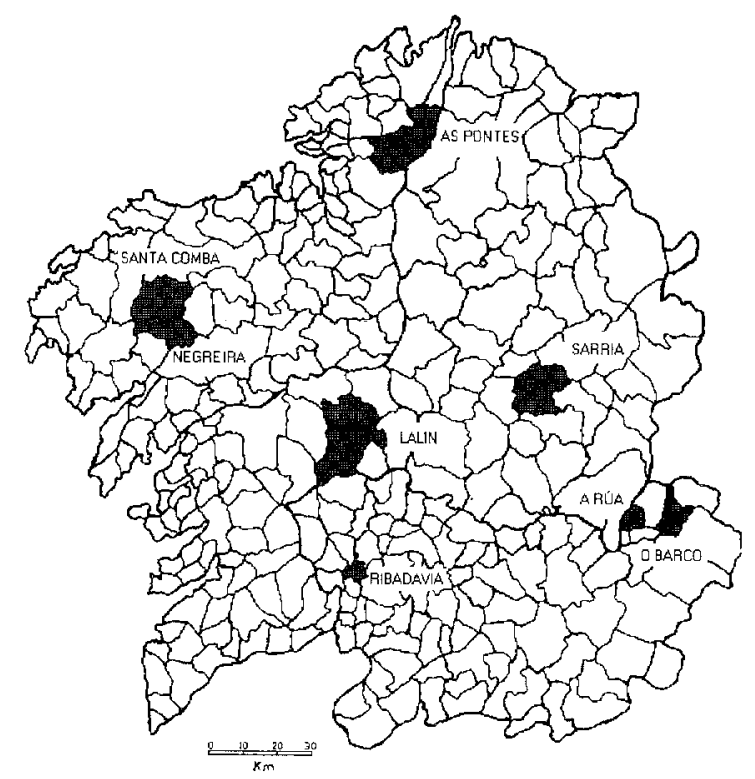

Figura 3.- Localización de los municipios de estudio. 
Superada esta fase de transición las tres últimas décadas consolidan el proceso iniciado anteriormente. Aquí la divergencia alcanza cuotas superiores observándose claramente un incremento superior en las villas respecto al total gallego, y sobre todo aquéllas vinculadas a procesos de industrialización endógena. Este contraste se entiende en función de la emigración y el trasvase de población agraria a otros sectores productivos. Ello supone que en los municipios en los que predomina el empleo agrario el decrecimiento sea generalizado (gran parte de Galicia) mientras en aquéllos que cuentan con puestos de trabajo en la industria, construcción o servicios, las variaciones sean positivas (LOIS GONZALEZ y RODRIGUEZ GONZALEZ, 1994).

Las razones básicas que nos ayudan a comprender este fenómeno, y que se relacionan directamente con el hilo argumental de nuestro trabajo, se basan en la constante ganancia de funcionalidad por parte de las villas y pequeñas ciudades de los espacios agrarios de Galicia. Estas, como ya expusimos con anterioridad, se basan en el impacto de diversos factores coetáneos en el tiempo: descentralización de servicios, inversión de remesas de capital procedentes de la emigración, transformaciones en las estructuras productivas agrarias,..., siendo el más destacable de todos el vinculado a procesos de industrialización endógena.

A partir de aquí se origina una red de pequeñas ciudades y villas localizadas de forma relativamente regular por el territorio y que sufren diversos procesos de especialización funcional en relación a la actividad económica en la que basan su desarrollo. Tanto es así que podemos establecer diferencias entre lo que entendemos por villas vinculadas a actividades ganaderas, vitícolas, mineras e industriales de carácter diversificado. Que presentan características demográficas, económicas, funcionales,... particulares. Complicando y dando una importante riqueza de matices a los estudios que abordan a las villas y pequeñas ciudades como centros de desarrollo local.

\section{Villas vinculadas a actividades agrícola/ganaderas}

- Villas del modelo de comarca ganadera

Galicia experimenta desde mediados de la presente centuria una creciente especialización pecuaria en beneficio de la producción láctea. Pero el impacto de este proceso se ha dejado sentir de forma desigual por el territorio, así se han configurado una serie de espacios comarcales donde la presencia de estas actividades es predominante en las estructuras productivas locales, y por otros donde adquiere una menor relevancia. Entre los primeros se encuentran los municipios de Santa Comba y Negreira en las tierras centrales de La Coruña. 
Con unos volúmenes demográficos modestos, Negreira 1.718 Hbt. y Santa Comba $1.683 \mathrm{Hbt}$. en 1991, estas villas van a beneficiarse del impacto de las importantísimas transformaciones ganaderas que se han dado en los últimos años. El paso de una economía agraria de autoconsumo y subsistencia a otra cuyas producciones están destinadas al mercado, va a traer consigo un importante cambio en las estructuras productivas y sociales del campo gallego (ITURRA, 1989). Este se traduce de forma inmediata en la capitalización de la producción agraria con la aparición de una serie de insumos que las explotaciones agrarias tienen que consumir en su proceso productivo y de una creciente mecanización de la actividad agraria.

Esto se traduce de dos formas directas. La primera se entiende en base a la transformación del agricultor que pasa de ser un ente productivo cuasi autárquico en la anterior fase, a una nueva situación en la que se ve inmerso en circuitos comerciales y productivos de ámbito internacional obligándole a una reconversión hacia nuevos esquemas más empresariales. En segundo lugar, estas transformaciones implican un excedente de mano de obra agraria que opta bien por la emigración fuera de la región bien por un trasvase de sector productivo.

Su papel hacia un desarrollo global se entiende en la función de complementación que desempeñan respecto a las crecientes necesidades de la actividad ganadera, dando lugar a la aparición de modestas empresas orientadas a cubrirlas. Nacen así, las fábricas de piensos, almacenes de redistribución de fertilizantes, talleres y fábricas de maquinaria agrícola, etc. Por otra parte, la existencia de un producto base susceptible de elaboración va a favorecer la aparición de otro tipo de empresas, esta vez dedicadas a transformar la producción obtenida: mataderos, elaboración de lácteos, etc., en un claro proceso de integración económica y aprovechamiento integral de los recursos endógenos.

La aparición de estas actividades complementarias y de aprovechamiento se liga fundamentalmente a la iniciativa de los propios productores mediante procesos asociativos y de formación de cooperativas tales como Feiraco, Leche Xallas o Xalco entre otras. Aunque tampoco podemos negar la introducción de capitales y empresas privadas, algunas de ámbito multinacional, en el sector agroindustrial (especialmente en el sector lácteo).

La importancia que desempeñan para un desarrollo integral del territorio no ha de medirse solamente por el empleo directo que generan, pues los valores para los dos municipios de empleo industrial no llegan al $20 \%$ de la población activa (la empresa que más trabajadores tiene es Feiraco con 223 en todas sus secciones), sino por la función de complementariedad que juegan y por la influencia indirecta en otros sectores de actividad: transportistas, talleres, etc. A modo de ejemplo Negreira tiene 172 licencias de transportista autónomo, Santa Comba cerca de 100. 
Sin embargo todas estas transformaciones y nuevas actividades, si bien favorecen la especialización funcional de estos núcleos, no permitirán unos ritmos de crecimiento demográfico similares a los otros ejemplos seleccionados para este estudio. Mostrándonos las limitaciones que presentan los modelos de desarrollo de las villas basados exclusivamente en la complementación de las actividades ganaderas.

- Villas del modelo de aprovechamiento de la vid

Dentro de Galicia las comarcas de El Ribeiro y O Valdeorras gozan de unas características propicias para la elaboración de vinos comercializables. Esta particularidad se ha traducido históricamente en la existencia de modestos núcleos urbanos que canalizaban los excedentes productivos. Prueba de ello es que las villas y pequeñas ciudades que centralizan estas comarcas gozaron tradicionalmente de cierto volumen demográfico. Así Ribadavia goza de un notorio pasado medieval y A Rúa, en Valdeorras, ya superaba los $2.000 \mathrm{Hbt}$ a principio de siglo. Este dato debe ponerse en relación tanto con los propios ritmos de trabajo que implica la vid, y que permite a los agricultores residir relativamente alejados de las tierras de cultivo, como por tratarse de comarcas tradicionalmente exportadoras de su producción, favoreciendo la aparición de grupos de personas dedicados a su comercialización. Gentes no relacionadas directamente con el trabajo en el campo y que podían residir en las villas cabeceras.

Pese a esta importante actividad, sus ritmos de crecimiento a lo largo del presente siglo (Ribadavia crece un $144 \%$ entre 1900 y 1991 y A Rúa un $248 \%$ ) han sido relativamente modestos. Si bien superan a las anteriores villas del modelo ganadero tanto en volumen total como en incrementos porcentuales, sin embargo son netamente inferiores a aquéllas que basan su desarrollo en una base industrial endógena.

Por otra parte, es destacable que en los ejemplos seleccionados se han consolidado funciones económicas paralelas. Así Ribadavia se especializa en la fabricación de ataudes, con un volumen de empleo directo que ronda los 300 trabajadores, y un total de 44 establecimientos dedicados a tal función (destaca pese a ello una empresa: Chao Sobrino con 97 empleados directos y una facturación que ronda los 500 millones de pesetas). Mientras, A Rúa se beneficia directamente de la centralización del control de producción y transporte de energía eléctrica del curso medio del río Sil, con la ubicación en su núcleo de la central de Iberduero. Y, también, de la creciente importancia que el sector pizarrero adquiere en toda su comarca.

Pero si los ritmos de crecimiento demográfico en el presente siglo se emparentan con las anteriores ramas productivas, es indudable que el cultivo y comercialización de la vid repercute directamente en las estructuras productivas de estas villas y sus comarcas. Así, como en el anterior caso, las pautas de comercialización arrastran la 
necesidad de fomentar el asociacionismo por parte de los productores minoristas para poder hacer frente a las exigencias del mercado (la empresa de mayor facturación del sector en Galicia es la Coop. Vitícola del Ribeiro, localizada en Ribadavia, con 761 millones en 1991). Mientras, algunos grandes cosecheros o bodegueros continúan, aunque con el lógico crecimiento de la producción, en régimen individual o bajo Sociedades Limitadas.

Los procesos de integración y de conexión de la base productiva local con los recursos tiene, también, su reflejo en la existencia de numerosas plazas de elaboración y distribución de vinos y derivados. En Ribadavia contamos con 42 unidades elaboradoras de corte semiartesanal e industrial, frente a las 7 de A Rúa. También se desarrollan servicios de apoyo al sector (en Ribadavia 18 licencias de venta mayorista de vinos) que favorecen su funcionamiento y aseguran un proceso integral.

\section{Villas vinculadas a actividades industriales}

- Villas del modelo minero

En Galicia se observan dos modelos diferenciados de desarrollo de pequeñas ciudades vinculadas a explotaciones mineras. Por una parte, el municipio de As Pontes (municipio con importantes reservas de lignito) tras la implantación de Endesa experimentará un espectacular incremento demográfico y funcional con nuevas actividades comerciales y de servicios orientadas a satisfacer las demandas y necesidades de los trabajadores de la mina y sus familias. Con 2.909 empleos industriales directos, $71,3 \%$ de la población activa total, podemos afirmar que nos encontramos ante un claro caso de monocultivo industrial del cual depende el resto de la estructura económica del municipio. Así se consolida un sector de empresas y servicios que conectan sus actividades con Endesa sirviéndole de complemento en su proceso productivo (importante presencia de empresas de transportes, ingeniería de montajes y construcción).

Por otra parte, en la comarca de Valdeorras se gesta un subsistema comarcal formado por emergentes asentamientos urbanos de los cuales el más importante es $\mathrm{O}$ Barco y, siguiéndole, A Rúa. la extracción y elaboración de Pizarra es la actividad reina aportando al sector cerca del $80 \%$ de la producción nacional. Sin embargo aquí la estructura empresarial es diferente al predominar pequeñas y medianas empresas con facturaciones inferiores a los 500 millones de pesetas.

De las 25 plantas extractoras con las que cuenta el sector pizarrero en Galicia, 20 se localizan en la comarca de Valdeorras lo que supone una importante oferta de empleo que alcanza sus cuotas máximas en el municipio de Barballeda donde el trabajo en la industria implica al $71,8 \%$ de la población activa total, siendo el 
porcentaje más alto de Galicia. O Barco, sin embargo, cuenta con unos niveles porcentuales más reducidos pues sus funciones terciarias derivadas de la categoría de pequeña ciudad que tiene (comercio, sanidad, educación,...) le permite tener una base económica más diversificada. No obstante, la dependencia de la actividad pizarrera es innegable y atiende de manera directa o indirecta a todo el contexto en el que se desarrolla la vida de la comarca del Valdeorras.

La relevancia de la actividad minera de cara al desarrollo de estas pequeñas ciudades es innegable. Permitiéndoles unos ritmos de crecimiento espectaculares respecto a 1900 (O barco $495 \%$ y As Pontes $642 \%$ ) y una población total que ronda los 10.000 Hbt. para 1991. Destaca, además, una acentuación de estos ritmos a partir de los sesenta, coincidiendo temporalmente con el afianzamiento de las empresas mineras. Lo que nos habla del paralelismo existente entre el desarrollo del aprovechamiento minero y el fortalecimiento de las villas que lo centralizan.

- Villas del modelo industrial diversificado

En este tipo de villas se produce una complementariedad de diferentes procesos de desarrollo. Así, de los ejemplos seleccionados tanto Sarria como Lalín, participan, al ser núcleos centrales de comarcas agrarias, de los procesos de especialización propios de las villas del modelo ganadero, presentando un sector agroindustrial comparable al de los ejemplos de Negreira o Santa Comba. Pero, además, han vivido otro tipo de procesos que les ha permitido consolidar una base productiva de carácter industrial basada en recursos endógenos. Permitiendo a sus núcleos urbanos crecimientos demográficos notables a lo largo del siglo XX (Lalín crece un $669 \%$ entre 1900 y 1991 y Sarria un 417 \%), especialmente a partir de los sesenta y alcanzar unos volúmenes que rondan los 7.000 Hbt. en 1991.

La base de este modelo de desarrollo se basa en el aprovechamiento de una serie de recursos endógenos no exclusivamente de carácter natural o físico, sino de carácter estratégico. De entre ellos destacan factores tales como la accesibilidad respecto a los núcleos rectores del sistema urbano, existencia de una cultura empresarial que beneficie la inversión de capital, posibilidad de contar con mano de obra abundante o favorable a relaciones contractuales informales. Toda esta gama de factores ha permitido establecer ventajas comparativas respecto a otras áreas productivas y fomentar la consolidación de un número significativo de pequeñas y medianas empresas altamente competitivas, y especialmente en un número restringido de sectores productivos.

De este discurso sólo se escapa, en el caso de Sarria, la empresa de cementos Cosmo. Planta química perteneciente a un grupo financiero portugués y que arroja unos volúmenes directos de empleo superiores a los 350 trabajadores. Pero exceptuando este 
caso puntual, la mayoría de las empresas de estos ejemplos se constituyen alrededor de capitales e iniciativas promovidas por agentes locales. Paralelo a esto en Sarria se han consolidado dos sectores industriales de desigual impacto en la base productiva propia. Por una parte se ha asentado en las últimas décadas la actividad vinculada a la construcción de muebles, con cerca de 300 empleos directos repartidos en cerca de 100 establecimientos, si bien es necesario insistir de nuevo en la existencia de un número restringido de plantas fabriles y en la paralela existencia de gran cantidad de talleres artesanales.

Por otra, y este sí es un recurso vinculado a riquezas naturales, alrededor de la planta de envasado de aguas minerales Fontecelta (45 trabajadores directos y facturaciones superiores a los 500 millones) se constituyen una serie de empresas que complementan su ciclo productivo y comercial (fábricas de envases de plástico, flotas de transportistas,...) en un claro proceso de integración y difusión económica.

Lalín, por el contrario, especializa su base empresarial en dos direcciones. En primer lugar, se detecta una creciente consolidación del sector textil y de la confección con 25 establecimientos y más de 350 trabajadores censados. En segundo, la carpintería metálica con más de 150 empleados. Ambas actividades tienen su punto de partida más en procesos de expansión estratégicos que en la explotación de recursos naturales.

Junto a esta secuencia sintetizadora de la estructura productiva industrial, debemos retomar la idea de que estos asentamientos tienen una base económica diversificada e inciden fuertemente en el desarrollo de su entorno ya que gran parte de los empleos industriales van a ser ocupados por personas que residen en el medio rural. Esta circunstancia explica tanto la aparición de movimientos pendulares casa-trabajo, como el bajo porcentaje de activos secundarios en ambos núcleos, 21,9\% de la población activa del núcleo de Lalín y $23,6 \%$ de Sarria, claramente inferiores a los 74,5 \% y 65,7 $\%$ que respectivamente se emplea en el sector terciario. Tal índice porcentual ha de relacionarse con el papel de centro comarcal de bienes y servicios y no debe desviar nuestra atención de la industria como principal factor de desarrollo de estos núcleos.

\section{BIBLIOGRAFIA}

ALDOMA BUIXADE, I. (1987): "Petites ciutats i desevolupament rural. El cas de la Catalunya interior", en Les ciutats petites $i$ mitjanes a Catalunya: evolució recent $i$ problemàtica actual, Institut Cartogràfic de Catalunya, Lleida. 
BEAUJEU-GARNIER, J. (Dir.) (1983): "Bilans regionaux ou departentaux de la descentralisation industrielle", en Cahiers du Centre de Recherches Analyse de l'Espace, 3-4, Paris.

DE VRIES, J. (1987): La urbanización en Europa 1500-1800. Ed. Crítica, Barcelona.

ITURRA, R. (1989): "Racionalidade tradicional, racionalidade individual: reciprocidade e optimizaçao nas estratexias productivas", en Actas do Il Coloquio de Antropoloxia, Xunta de Galicia, Santiago de Compostela.

LABORIE, J.P. (1991): L'avenir des petites villes, C.I.E.U., Toulouse.

LOIS GONZALEZ, R.C. (1993): "Problemas para a delimitación dos espacios urbanos e rurais", en Concepcions espaciais e estratexias territoriais na Historia de Galicia, A.G.H. Santiago de Compostela.

LOIS GONZALEZ, R.C. y RODRIGUEZ GONZALEZ, R. (1994): "O retroceso da vida campesiña e a crise da vida parroquial en Galicia. A importancia da análise microespacial", en Revista de Investigación Pontenova, Diputación de Pontevedra.

McLOGHLIN, J.B. (1971): Planificaciónurbana y regional. Un enfoque de sistemas. I.E.A.L., Madrid.

PEREZ VILARIÑO, J. (1990): "As cidades alineadas do Atlántico e a economia política metropolitana en Galicia", en Revista Galega de Economia, 0, Santiago de Compostela.

PRECEDO LEDO, A. y MARTINEZ CONDE, R. (1991): "El modelo de transición urbana en un área periférica", en Geéographie Sociale, 11, Université du Mans, Caen.

RIBAS Y PIERA, M. (1974): Estudios de Economía Urbana. I.E.A., Madrid.

RODRIGUEZ GALDO, M.J., PIS SANCHEZ, E. y COSTA CAMPI (1992): Evaluación del potencial endógeno de Galicia. IDEGA, Santiago de Compostela.

RODRIGUEZ GONZALEZ, R.: Lalín, estudio de Xeografía Urbana. Memoria de Licenciatura, (en prensa).

SEQUEIROS TIZON, J.G. (1986): El desarrollo económico en Galicia. Universidad de Santiago de Compostela, Santiago de Compostela.

SOUTO GONZALEZ, X.M. (1988): Xeografía Humana, Ed. Galaxia, Vigo.

VAZQUEZ BARQUERO, A. (1988): Desarrollo local. Ed Pirámide, Madrid. 
VINUESA ANGULO, J. y VIDAL DOMINGUEZ, M.J. (1991): Los procesos de urbanización. Ed. Síntesis, Madrid.

XUNTA DE GALICIA (1993): Directorio Industrial de Galicia. Conselleria de Economía, Santiago de Compostela. 